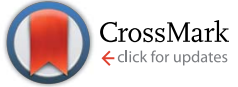

Cite this: RSC Adv., 2015, 5, 60786

Received 30th June 2015 Accepted 7th July 2015

DOI: $10.1039 / \mathrm{c} 5 \mathrm{ra12684c}$

www.rsc.org/advances

\section{Preparation and rheological properties of whey protein emulsion fluid gels}

\author{
R. J. A. Moakes, ${ }^{*}$ A. Sullo and I. T. Norton
}

A shear-gel approach was used to coat an O/W emulsion with a whey protein shell. Mechanical shear was applied to an aqueous solution of whey protein isolate (WPI) and oil then heated through the sol-gel transition. The formation of a continuous WPI network was prevented through the shear flow, resulting in discreet spherical capsules, with sizes in the micron scale $(\sim 25 \mu \mathrm{m})$. Through careful control over both the oil and WPI components, encapsulation efficiencies of up to $99 \%$ were obtained. Subsequent rheological properties highlighted elastic behaviour $\left(G^{\prime}\right)$ dependent on oil content; where higher oil fractions increased the effective phase volume of the particles. The result was packing fractions that exceeded those for hard spheres (>0.64), leading to pseudo-solid characteristics at rest, apparent yield stresses and thixotropic behaviour under shear. As such, emulsion fluid gel (EmFG) rheology was closely comparable to those of soft microgel suspensions (fluid gels) and soft-coated particles.

\section{Introduction}

The use of particulates as rheological modifiers spans across multiple industries: paint, cosmetic, food etc. For this reason, systems such as colloidal suspensions and emulsions have been well documented. However, more recently microparticulate gel suspensions have received increasing interest for their ability to create weakly structured fluids, with rheological properties characterised between those for colloidal particles and polymeric gels; pseudo-solid behaviour at rest, but flow above a critical stress. ${ }^{1-4}$ Enhanced flow behaviours have been observed in polysaccharide fluid gels where, at volume fractions as low as $\phi 0.2$ suspensions showed high viscosities and marked shear thinning behaviour, typical of highly aggregated systems. ${ }^{5,6}$ The observed changes in flow properties were assigned to the particle microstructures, where, as a result of incomplete gelation at the particle surface, interactions between microgel spheres resulted in a degree of structuring. Additionally, the deformability of such soft hydrogel particles led to volume fractions that exceeded those typical for hard spheres. At such high volume fractions particles become sterically confined, thus rheology became more closely governed by the particle moduli. ${ }^{7-9}$

Particle intrinsic properties such as strength and deformability can be better understood by looking at a comparable quiescently formed gel. Again, mechanical properties are closely linked to the gel microstructure. ${ }^{\mathbf{1 0 - 1 4}}$ Complex microstructures therefore provide another means of controlling a gel's physicomechanical properties. Besides polymer mixes where two or

Centre for Formulation Engineering, School of Chemical Engineering, University of Birmingham, Edgbaston, Birmingham,B15 2TT, UK.E-mail: rjm116@bham.ac.uk more polymers undergo a sol-gel transition, filler particles can be used to prepare gel composites. ${ }^{15}$ These composites display viscoelastic properties as a function of the gel matrix, volume fraction and rigidity of the filler. Chemical affinity between the filler and surrounding substrate is also very important. ${ }^{16}$ Typically, where the filler interface is included into the gelled network strengthening of the matrix occurs. ${ }^{17}$ It was demonstrated that increasing the time scale between emulsification and gelation resulted in a shift from stronger to weaker gels. Here, polymerisation of the emulsifier lowered the affinity between oil filler and substrate matrix, causing inherent weak spots within the gel. ${ }^{17-19}$

Processing is therefore a major consideration in the preparation of gels, on both a macro and micro-scale. Microgels are typically formed by confining the polymer during the sol-gel transition. This can be achieved by means of chemical $^{20}$ or mechanical $^{\mathbf{1}}$ separation. Controlled microstructures and further rheological properties can be achieved by a microstructural design approach; whereby changing the formulation, i.e. composition, degree of cross-linking and system $\mathrm{pH}$, leads to careful control over the gelled network. ${ }^{\mathbf{4} 21}$ Additionally, in the case of shear gels, modulating the two processing parameters shear and thermal history can lead to controlled particle size and morphology. When the separation applied to the system is such that it becomes comparable to the timescale for polymer ordering, random coil to helix transition and subsequent gelation through the formation of junction sites, thermodynamically favourable spherical particles $(\sim 1 \mu \mathrm{m})$ are formed: where the enthalpies of melting are much smaller to comparable quiescently prepared gels. ${ }^{22}$ The rate of particle growth therefore becomes key. Large anisotropic $(\sim 100 \mu \mathrm{m})$ particles form when structuring is so rapid that initial gels are able to form, 
becoming subsequently broken down in the shear flow. However, in whey protein systems an inverse relationship between aggregation kinetics and system elasticity was observed, showing faster rates to yield weaker suspensions, with lower yield stresses and viscosities. ${ }^{23}$

Supramolecular chemistry has also shown a viable route to the preparation of micro-composites. ${ }^{24-26}$ These particles are formed through electrostatic bonding of the polymers. The particulates are formed by carefully controlling the $\mathrm{pH}$ so that the zeta-potential of both polymers is of an opposite charge, with a potential great enough to drive complexation. Such micro-particulates, based on an $\mathrm{O} / \mathrm{W}_{1} / \mathrm{W}_{2}$ system, exhibited a several fold increase in viscosity when compared to a simple $\mathrm{O} / \mathrm{W}$ emulsion. ${ }^{24}$ These viscosity changes were attributed to a change in the effective phase volume of the hydrogel spheres, which were further increased through the inclusion of an oil filler. However, interactions between the particles were not observed.

We report here the preparation of emulsion fluid gel (EmFG) particles, a micro-composite of whey protein gel and oil. The research builds on work presenting $\mathrm{O} / \mathrm{W}_{1} / \mathrm{W}_{2}$ filled hydrogel systems by applying a "shear gel approach" to promote interactions between resulting particles. As such, it takes an additional step to surfactant or Pickering stabilised emulsions by gelling a continuous WPI layer around an oil core. The technique applied results in an elastic suspension, whereby the particles become trapped, suspended in an aqueous phase. Unlike similar studies ${ }^{27}$ the final systems act in a pseudo-solid fashion until a great enough stress is applied to induce flow, thinning the suspension into a liquid-like state. The microparticles thus offer the capacity to act as a multifunctional composite, for both controlled rheological applications and pose the potential to encapsulate poorly soluble molecules.

The work focuses on whey protein isolate (WPI) as the coating material owing to its thermo-denaturation and subsequent hydrophobic aggregation, to form a gel layer on the surface of an oil substrate. Shear separation will then be applied to prevent complete gelation, promoting particle-particle interactions. Therefore the production of emulsion fluid gels has been investigated, with particular attention to the resulting rheological properties.

\section{Materials and methods}

\subsection{Materials}

Whey protein isolate (WPI) was obtained from Kerry Ingredients, Listowel, Ireland (WPI, W994, S-493391) and used without further purification. WPI composition as stated by the supplier was $91.0 \%$ protein, moisture $4.0 \%$, fat $1.0 \%$, ash $3.5 \%$ and lactose $0.5 \%$. Mineral content of the WPI was: $\mathrm{Ca}-0.50, \mathrm{P}-$ $0.65, \mathrm{Na}-0.10, \mathrm{~K}-0.15, \mathrm{Mg}-0.02$ and $\mathrm{Cl}-0.02 \%$. High oleic sunflower oil was obtained from Cargill (Cargill Inc., BE). Sodium azide, hydrochloric acid, silicon oil, Nile Red and Rhodamine B were purchased from Sigma-Aldrich (SigmaAldrich, UK).

\subsection{Preparation of oil filled fluid gels}

Preparation of the oil filled fluid gels involved a three-step process. Primary solutions of WPI were firstly prepared and used to form $\mathrm{O} / \mathrm{W}$ emulsions. The emulsions were then heat treated under shear conditions resulting in emulsion fluid gels (EmFG).

2.2.1. Preparation of WPI stock solutions. Whey protein stock solutions (5 to $30 \mathrm{wt} \%$ on a protein basis) were prepared by dispersing WPI in deionised water. An anti-microbial, sodium azide $(0.02 \mathrm{wt} \%)$ was added to all solutions to enhance storage times. Solutions were stirred overnight at ambient conditions until completely hydrated and stored at $5{ }^{\circ} \mathrm{C}$ until further usage. For the preparation of stained samples Rhodamine B $(0.015 \mathrm{mM})$ was added to stain the protein. Stained samples were kept covered to prevent photo bleaching.

2.2.2. Preparation of emulsions. Oil in water emulsions were prepared by the addition of high oleic oil to WPI primary solution (protein concentration ranging from 5 to $30 \mathrm{wt} \%$ ) so that total volumes resulted in oil fractions ranging from 5 to 20 vol\%. The mixtures were subsequently mixed in a high shear mixer (Silverson, SL2T) at $4000 \mathrm{rpm}$ for 10 minutes. System pH was adjusted to $\mathrm{pH} 4.6$ with concentrated hydrochloric acid (12 M) and aged for 72 hours before gelling under shear. Stained EmFG were prepared with the addition of Nile Red $(0.015 \mathrm{mM})$ to the oil phase and kept covered to prevent photo bleaching.

2.2.3. Preparation of oil filled fluid gels. A jacketed vessel and overhead stirrer equipped with pitched blade impeller was used to prepare all WPI emulsion fluid gels (EmFG). Aliquots of emulsion were added to a jacketed vessel set to $50{ }^{\circ} \mathrm{C}$, controlled through a circulating water bath. Shear was applied through the stirrer and impeller at $450 \mathrm{rpm}$. Once thermal equilibrium was obtained ( $c$. 10 minutes), emulsions were heated at a rate of $0.5^{\circ} \mathrm{C} \min ^{-1}$ to $80{ }^{\circ} \mathrm{C}$. Suspensions were subsequently decanted and left to cool quiescently under ambient conditions. Cooled EmFG were further stored at $5{ }^{\circ} \mathrm{C}$ for seven days until rheologically tested. In all experiments a cover was applied to minimise water loss. When staining, the system was covered in aluminium foil to avoid fluorescence quenching.

\subsection{Static light scattering (SLS)}

A MS2000 Mastersizer with attached Hydro SM manual small volume dispersion unit (Malvern Instruments Ltd, UK) was used to obtain size distributions for EmFG particles. Distributions are the average of three repeats. Particle size calculations were based upon the Mie theory, thus particles were assumed to be monodisperse, homogenous spheres. Additionally, once coated, particles were a binary system of protein and oil; as such the refractive index of the shell was used.

\subsection{Microscopy}

2.4.1. Optical light microscopy. Samples were prepared by first diluting the EmFG in deionised water (7.5 vol\%). A Brunel SP300-fl (Brunel Microscopes Ltd, UK) optical light microscope fitted with an SLR camera (Cannon EOS Rebel XS, DS126 191) at 20 and $40 \times$ optical magnification was used to image the 
particles. Slides were prepared by addition of $50 \mu \mathrm{L}$ of sample to a microscope slide (VWR, UK) and covered with a coverslip (thickness no.1, VWR, UK).

2.4.2. Confocal laser scanning microscopy (CLSM). Stained samples prepared with both Rhodamine B and Nile Red were imaged using concave microscope slides $(60 \mu \mathrm{L})$, with a coverslip sealed using super glue. Fluorescence free, UV transparent immersion oil (Sigma-Aldrich, UK) was used to bridge the gap between coverslip and objective lens $(40 \times$ magnification). A Leica TCS-SPE confocal microscope (Leica Microsystems Ltd, UK) fitted with an argon laser was used for all CLSM analysis. Rhodamine B stained protein was excited at $532 \mathrm{~nm}$ and detected at 560-600 nm, and Nile Red was excited at $488 \mathrm{~nm}$ and detected at 680-700 $\mathrm{nm}$ respectfully. 3-Dimensional images were obtained using $0.5 \mu \mathrm{m}$ slices throughout. Further image processing was undertaken using an image software package (ImageJ).

\subsection{Rheological analysis}

Rheometry was conducted using a Bohlin Gemini HR Nano stress-controlled Rheometer (Malvern Instruments Ltd, UK) equipped with serrated parallel plate ( $25 \mathrm{~mm}$ diameter) at $1 \mathrm{~mm}$ gap height. Experiments were undertaken at $25{ }^{\circ} \mathrm{C}$ using a silicon oil moisture trap. An equilibrium was achieved for 15 minutes prior to testing, allowing for consistent loading conditions. Particle phase volumes were obtained using a method outlined by Moakes, et al. $;{ }^{23}$ whereby aliquots of a given volume were centrifuged, water phase separated and volumes obtained. Phase volumes were calculated as followed (eqn (1)):

$$
\text { Particle phase volume }=1-\left(\frac{\text { volume of water removed }}{\text { total initial volume }}\right)
$$

2.5.1. Yield stress determination. Yield stresses were determined as the stress at which the transition from storage modulus $\left(G^{\prime}\right)$ to loss modulus $\left(G^{\prime \prime}\right)$ dominance occurred; the $G^{\prime} / G^{\prime \prime}$ cross over point on a stress controlled amplitude sweep. Amplitude sweeps were conducted at $1 \mathrm{~Hz}$ from 0.1 to $400 \mathrm{~Pa}$.

2.5.2. Dynamic oscillatory measurements. Frequency sweeps were obtained between 0.1 and $10 \mathrm{~Hz}$ at controlled stress. The stress was determined by amplitude sweeps as a value within the linear viscoelastic region (LVR) for all EmFG tested. For samples containing no oil a, couette, double gap geometry was used: as the large surface area allowed lower values of $G^{\prime}$ to be probed in the lower viscosity fluids.

2.5.3. Viscosity measurements. Dynamic viscosity measurements were undertaken between 0.1 and $400 \mathrm{~s}^{-1}$. Shear sweep time was set to 10 minutes (ramp ascending and descending). Two consecutive sweeps were run with the second commencing immediately after the first having been completed.

2.5.4. Recovery analysis. EmFG recovery was probed by primarily rejuvenating the system. A pre-shear $\left(10 \mathrm{~s}^{-1}\right.$ for $\left.10 \mathrm{~s}\right)$ was applied to the system and subsequent change in storage modulus $\left(G^{\prime}\right)$ recorded over the following $30 \mathrm{~min} . G^{\prime}$ was obtained at $1 \mathrm{~Hz}$ and $1 \mathrm{~Pa}$.

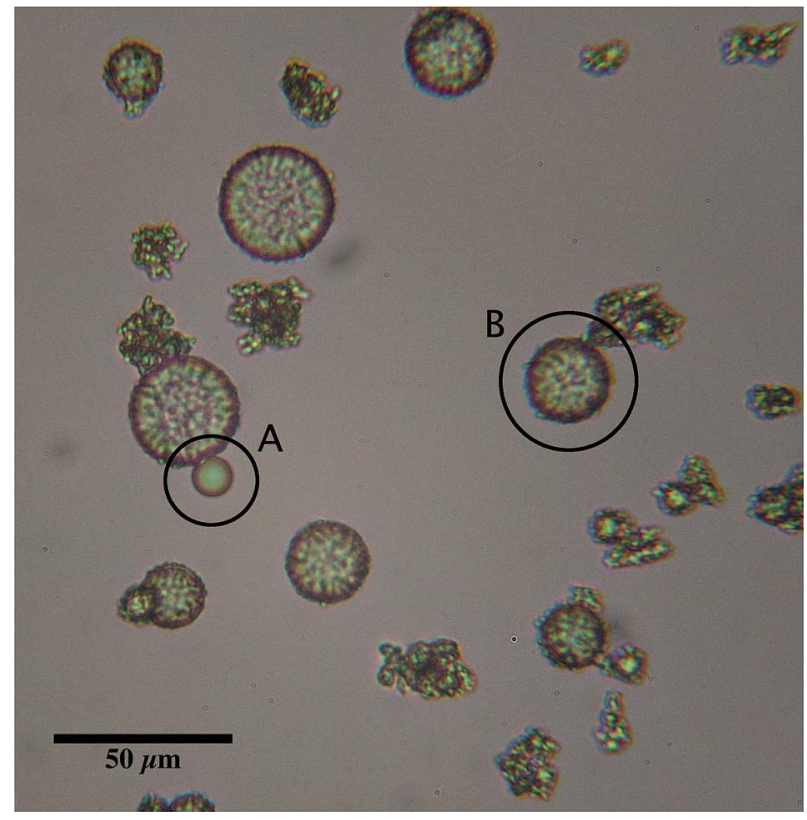

Fig. 1 Optical micrograph highlighting (a) an uncoated oil droplet and (b) a coated EmFG particle.

\subsection{Encapsulation efficiency}

Optical microscopy was used to analyse oil droplet entrapment for each system. Micrographs of the raw emulsion were obtained and emulsion droplets in each image manually counted to give an average droplet count $\left(N_{\mathrm{em}}\right)$ determined over 12 images. Values for uncoated emulsion droplets $\left(N_{\mathrm{fo}}\right)$ in the final EmFG systems were then obtained in the same manner. An example of both a coated and uncoated droplet has been shown in Fig. 1. The ratio of the two was used to calculate the percentage of encapsulated oil, as shown in eqn (2):

$$
{ }^{\circ}{ }_{\text {Encapsulated }}=\left(1-\frac{N_{\mathrm{fo}}}{N_{\mathrm{em}}}\right) \times 100
$$

Encapsulation was averaged over 12 micrographs with error calculated as the $95 \%$ confidence interval.

\section{Results and discussion}

\subsection{Preparation of emulsion fluid gels (EmFG)}

An $\mathrm{O} / \mathrm{W}$ system where the excess WPI emulsifier exceeded the critical gelling concentration ${ }^{28}(>1 \mathrm{wt} \%)$, was subjected to heat treatment under shear conditions. The shear flow exerted on the system during the sol-gel transition prevented the formation of a continuous gel network, resulting in single discreet particles/encapsulates.

3.1.1. Encapsulation efficiency. Fig. 2 shows the encapsulation efficiencies for systems with both increasing WPI concentration (a) and oil phase volume, $\phi_{\text {oil }}$, (b). It was observed that high levels of encapsulation, $\sim 99 \%$, were achieved in systems with lower WPI concentrations, however as the WPI exceeded $20 \mathrm{wt} \%$, entrapment decreased. It is argued that the 
degree of encapsulation is closely correlated to the flow behaviour of the system during the sol-gel transition. It has previously been reported that around the isoelectric point of WPI (ca. pH 5) a transition from Newtonian to pseudoplastic flow occurs in systems containing $20 \mathrm{wt} \%$ whey protein, with yield stresses observed on further concentrated solutions. ${ }^{29,30}$ Such changes in the system viscosity retard or prevent the diffusion of denatured protein to the oil/water interface due to a reduction in mobility and increased collisions with other denatured polymer chains. In turn, both increased formation of WPI aggregates without included oil and free emulsion droplets were observed in the final suspensions.

Increasing the phase volume of the oil from 5 to $20 \%$ whilst retaining a standard WPI concentration (15 wt\%) however, had little effect on the emulsion entrapment, yielding droplet entrapment in excess of $95 \%$. Here, the increase in oil was not sufficient to raise the viscosity of the system, and hinder polymer diffusion. However, at low oil fractions increasing the WPI concentration from 5 to $15 \mathrm{wt} \%$ caused an observed transition from suspension creaming to sedimentation, indicating a change in particle density, probably as a result of a change in shell thickness.
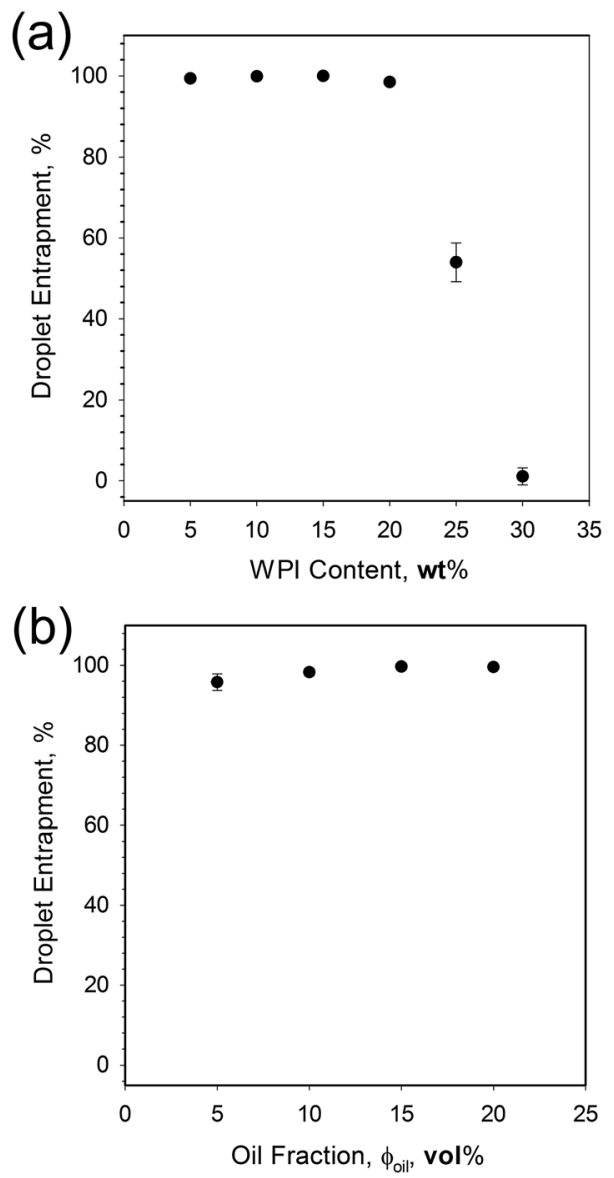

Fig. 2 Oil droplet entrapment efficiencies for (a) EmFG systems prepared with increasing WPI concentrations ranging between 5 and 30 wt $\%$ at a fixed $\phi_{\text {oil }}$ of 10 vol\%, and (b) EmFG systems prepared with increasing $\phi_{\text {oil }}$ between 5 and 20 vol\% with a fixed WPI concentration of $15 \mathrm{wt} \%$.
3.1.2. Particle morphology. Particle morphology was studied using confocal laser scanning microscopy (CLSM). Previous reports regarding the formation of WPI microgel particles through the application of shear, ${ }^{23}$ show irregular shaped particles, characterised by a larger length to width ratio. However, the incorporation of oil in to the system resulted in particles with spherical morphology, whereby a gel layer surrounded an oil substrate, as shown in Fig. 3.

Imaging the EmFG particles at $0.5 \mu \mathrm{m}$ intervals gave enhanced topographical detail (Fig. 3a), allowing the coating to be observed. A non-uniform shell with much greater thickness than expected for emulsified droplets was shown, inferring the presence of a gel layer. Additionally, cross-sections were obtained using CLSM, which again show shell thickness and nonuniformity, but also through negative staining and dying, oil reservoirs in the centre of the particles (Fig. $3 \mathrm{~b}$ and c respectfully).

The mechanism for particle formation is thought to be based upon the oil acting as a substrate for shell growth. Growth occurs through enrichment from the surrounding un-gelled biopolymer. Primarily led by the $\beta$-lactoglobulin, heat induced denaturation of the native structure causes hydrophobic regions to become exposed. ${ }^{31,32}$ Hydrophobic interactions then dominate the gelation causing diffusion of the denatured polymer to the oil/water interface. The oil droplet thus acts as a point for nucleation and growth. Shear imposed upon the system then restricts particle-particle aggregation, preventing a continuous network from forming. As a result, particles grow to an extent permitted by the shear flow, however are primarily dictated by the size of the emulsion droplets.

3.1.3. EmFG particle size distributions. Particle size distributions for all EmFG systems are shown in Fig. 4. It is clear from Fig. 4a that increasing WPI concentration results in a shift towards smaller particle sizes. Particles are primarily a function of the emulsion droplet size, thus such observations would be expected as increasing the emulsifier concentration causes the formation of smaller droplets. ${ }^{33,34}$ However, by further increasing the protein concentration up to $30 \mathrm{wt} \%$, the formation of a bimodal system centred at $\sim 9 \mu \mathrm{m}$, with a second peak at much higher particle sizes $(\sim 200 \mu \mathrm{m})$ was observed. The shift from monomodal to bimodal is due to the formation of gelled particles where no oil has been entrapped as observed through microscopy. As previously described, the change in system flow results in aggregated protein, as diffusion of the denatured polymer becomes restricted, causing a transition from proteinsubstrate to protein-protein interactions. The extent of such a transition is thus reflected by the change in peak intensity observed in systems with 20 to $30 \mathrm{wt} \%$ WPI.

Fig. $4 \mathrm{~b}$ shows little effect on the resulting particle size as a function of oil fraction, as a result of consistent emulsifier concentrations and unrestricted diffusion of biopolymer to the substrate interface. Thus, all distributions are centred around 25-30 $\mu \mathrm{m}$ complimenting sizes observed via microscopy (Fig. 1 and 3).

\subsection{EmFG material properties}

3.2.1. Small deformation oscillation testing. To further understand and characterise the EmFG physico-mechanical 

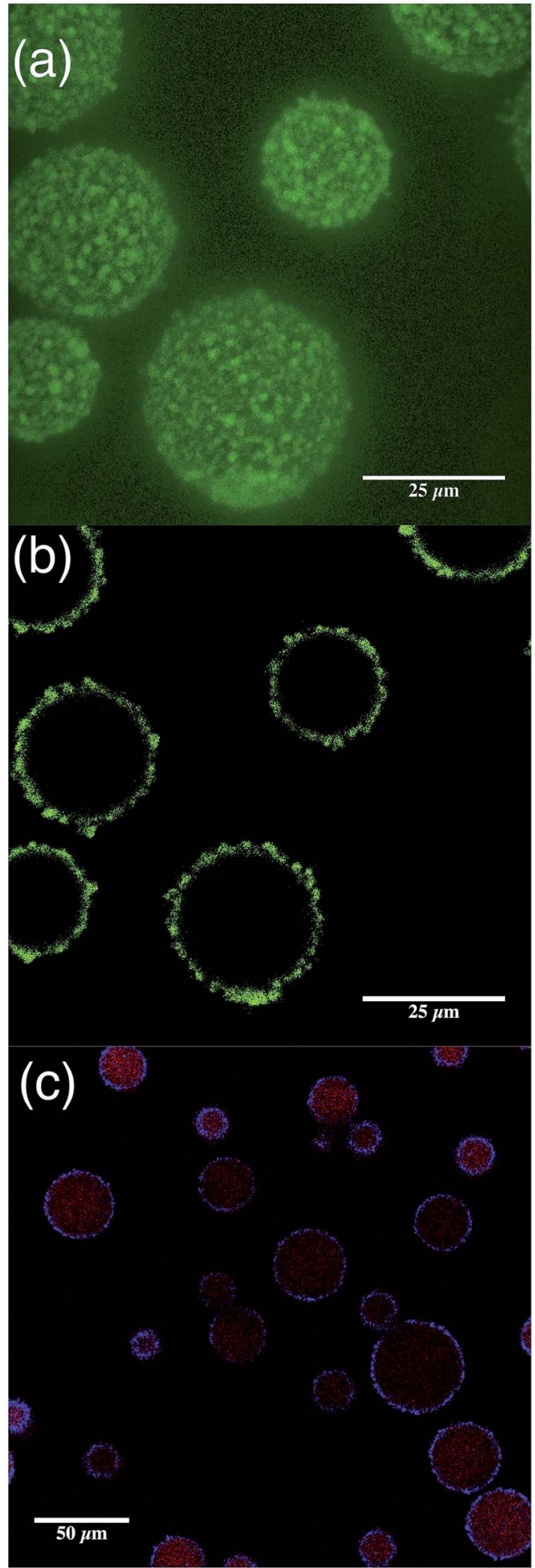

Fig. 3 CLSM micrographs of EmFG particles. Gel phase has been stained using Rhodamine b (excitation wavelength: $532 \mathrm{~nm}$, emission wavelength: $560-600 \mathrm{~nm}$ ) and oil phase either negatively stained or stained using Nile Red (excitation wavelength: $488 \mathrm{~nm}$, emission wavelength: 690-700 nm). (a) 3D stack showing topographical detail of the EmFG particles, (b) cross section depicting stained gel layer surrounding a negatively stained oil core, and (c) stained cross section showing protein shell (blue) and oil core (red). Scale bars represent 25 $\mu \mathrm{m}(\mathrm{a}$ and $\mathrm{b}$ ) and $50 \mu \mathrm{m}$ (c). properties, small deformation rheology was carried out. Fig. 5a shows frequency sweeps obtained for EmFG systems prepared with varying oil fractions from 0 to 20 vol\%. Systems containing no oil displayed typical liquid-like behaviour where both moduli were dependent on frequency and $G^{\prime \prime}$ is higher than $G^{\prime}$ throughout the measured frequency range. ${ }^{35}$ The addition of oil caused a transition in rheological behaviour to pseudo-solid where $G^{\prime}$ is higher than $G^{\prime \prime}$, with both moduli becoming further independent to frequency as a function of the oil. Here particle proximity is such that inter-particle interactions arise as observed for WPI microgel particles, ${ }^{23}$ however, as the shear and thermal history exerted was consistent across all systems, it is argued that system elasticity becomes a function of the oil content; where increasing oil fractions increase the effective phase volumes of the particles, as shown in Fig. 5 b.

To better understand the mechanism through which system elasticity arises, the results obtained have been compared to models already proposed for particulate suspensions. The Krieger-Dougherty (KD) model is used to describe the relationship between relative viscosity $\left(\eta_{\text {rel }}\right)$ and particle phase volume for hard sphere suspensions, ${ }^{36}$ eqn (3).

$$
\eta_{\text {rel }}=\left(1-\frac{\phi}{\phi_{\max }}\right)^{-[\eta] \phi_{\max }}
$$

The equation relates the ratio between the phase volume of the suspension, $\phi$, to the maximum packing fraction for monodisperse hard spheres (0.64), $\phi_{\max }$, as a function of the intrinsic viscosity, $[\eta]$. What is clear from eqn (3) is that as the maximum packing fraction is approached the relative viscosity will asymptote and eventually the equation fail as the suspension reaches/surpasses the maximum packing. As such, the KD equation cannot be used to describe the correlation observed in Fig. 5b, where the maximum packing fraction for hard spheres has been exceeded.

Similar observations have been reported for agar microgel suspensions, ${ }^{9}$ where above a critical volume fraction, $\phi_{\mathrm{c}}$, elastic response was observed, becoming insensitive to phase volumes above $\phi_{\max }$. It was explained that above $\phi_{c}$, elasticity was a function of the particle modulus; hence particles were typically acting as soft spheres, however the plateauing effect was left unexplained, suggested as an artefact of the phase volume calculation. EmFG are therefore assumed to act as soft spheres. It is suggested that the soft oil core and elastic whey protein shell allows for particle deformation when highly concentrated, reaching phase volumes that exceed those expected for rigid spheres. At such high phase volumes a jamming phenomenon is observed, thus particle rheology mainly represents a function of the shell. ${ }^{37}$ Hence above a volume fraction of 0.64 particles are packed to an extent that system elasticity is close to those shown for filled quiescent gels, ${ }^{38}$ hence frequency sweeps show gel-like behaviour (Fig. 5a).

EmFG prepared using 5 vol\% oil showed marked storage moduli even though $\phi_{\max }$ had not been reached. Here, elastic 
(a)

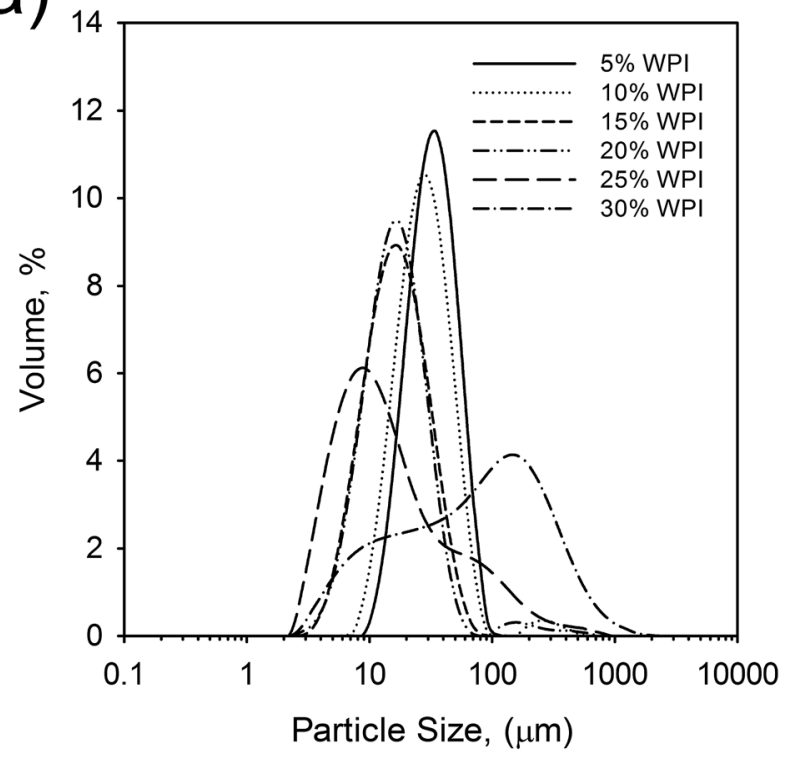

(b)

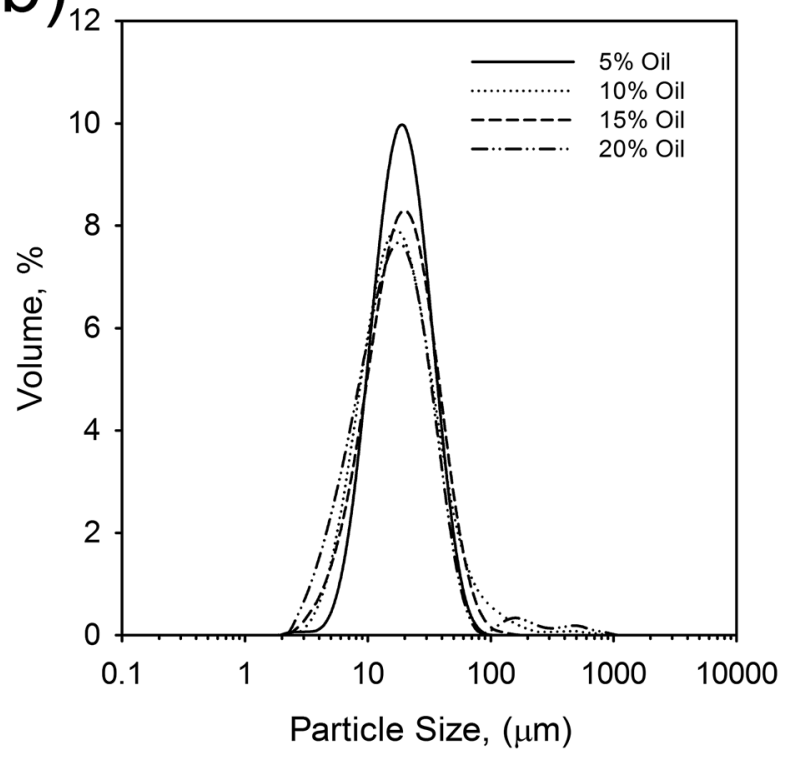

Fig. 4 Particle size distributions for (a) EmFG prepared with a range of WPI concentrations between 5 and 30 wt\% with a fixed $\phi_{\text {oil }}$ of 10 vol\%, and (b) EmFG prepared with a range of $\phi_{\text {oil }}$ from 5 to 20 vol\% with a fixed WPI content of $20 \mathrm{wt} \%$.

response is observed not through the jamming of particles, but assumed to arise from particle trapping as a result of steric confinement through inter-particle interactions. Between $\phi_{\mathrm{c}}$ and $\phi_{\max }$ it is argued that EmFG act as a glass where particles become trapped allowing localised motion but not long range diffusion, similarly to results published by Koumakis, et al. ${ }^{37}$ and le Grand. ${ }^{39}$ Therefore, the EmFG can be categorised into three regimes; suspended particles below $\phi_{\mathrm{c}}$, glassy between $\phi_{\mathrm{c}}$ and $\phi_{\max }$, and jammed above $\phi_{\max }$.

3.2.2. Material yielding behaviour. The effect of oil fraction on material response was further probed through the use of (a)

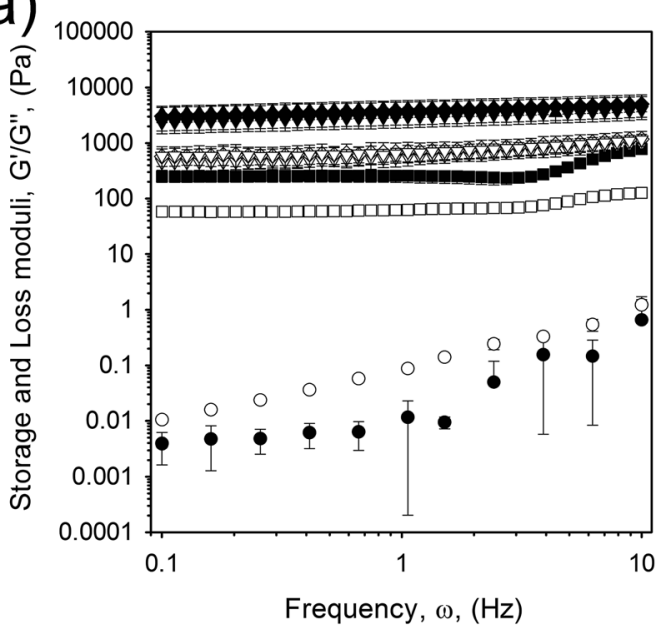

(b)

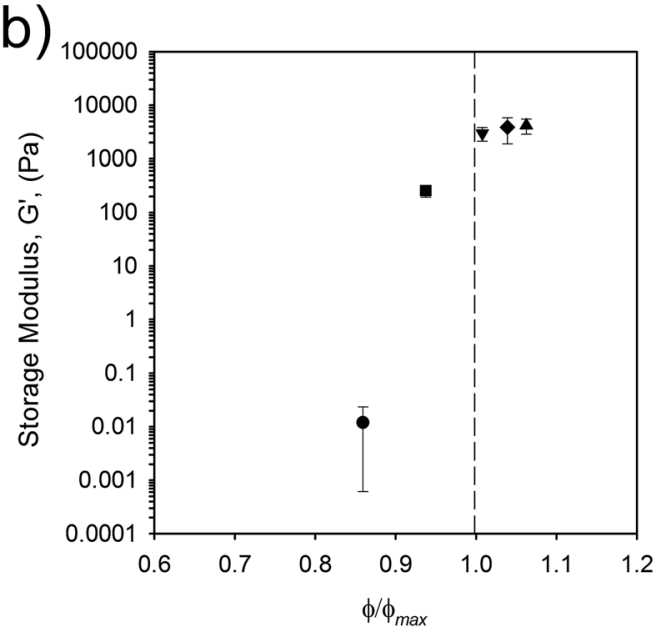

Fig. 5 (a) Frequency sweeps obtained for EmFG prepared using 20 wt\% WPI and $(\boldsymbol{O}, 0)$ no oil, $(\boldsymbol{\square}, \square) 5$ vol\% oil, $(\boldsymbol{\nabla}, \nabla) 10$ vol\% oil, $(\diamond, \diamond) 15$ vol\% oil, and $(\boldsymbol{\Delta}, \triangle) 20$ vol\% oil. Open markers represent the storage moduli $\left(G^{\prime}\right)$ and closed show loss moduli $\left(G^{\prime \prime}\right)$. (b) Storage modulus at $1 \mathrm{~Hz}$ verses particle phase volume for systems containing

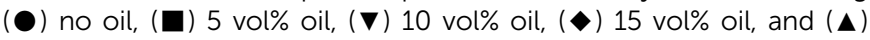
20 vol\% oil. Dashed line represents maximum packing faction for hard spheres.

stress sweeps. Fig. 6 shows the data obtained for EmFG with increasing oil content (from (a) to (d)). The stress sweeps indicate that at a critical stress the network started to break down. Further increasing the stress led to a transition at which point the loss modulus dominated the storage. At this point a change in material response occurs, where the system no longer resembles a pseudo-solid, but is much more fluid-like. For systems in a glassy state, where particle have become trapped through inter-particle interactions, the linear viscoelastic region (LVR) was observed to be much shorter than those that are jammed. Again, such observations can be interpreted through the deformability of the particles; where particles are in close proximity they become compressed and deform, a larger stress is needed to induce flow as observed for $\kappa$-carrageenan fluid gels. ${ }^{6}$ However, for systems where $\phi$ does not surpass $\phi_{\max }$, 
(a)

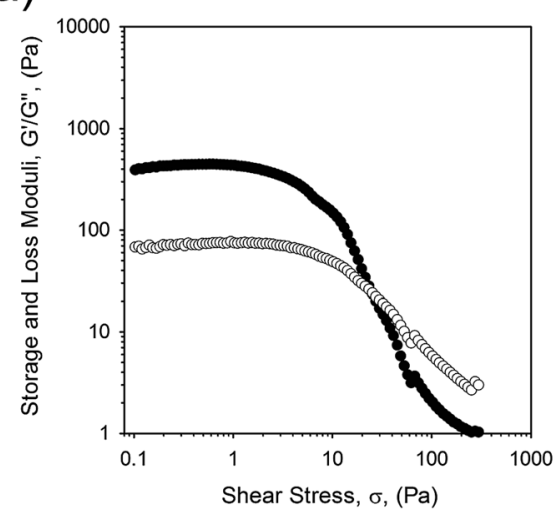

(c)

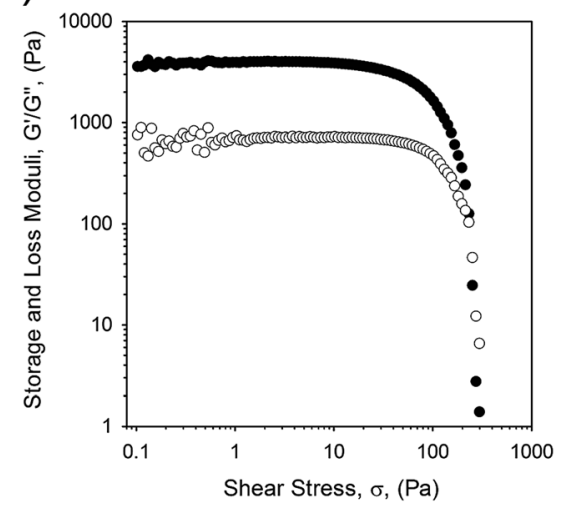

(b)

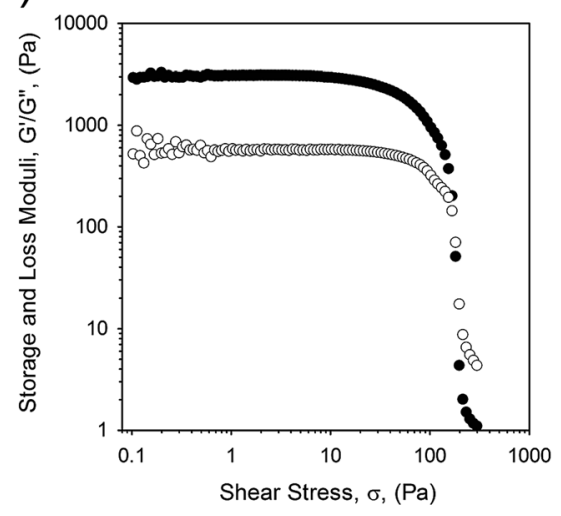

(d)

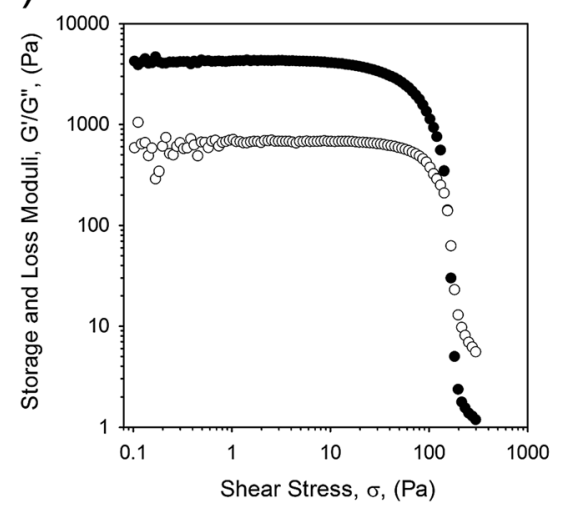

Fig. 6 Stress sweeps obtained at $1 \mathrm{~Hz}$ for EmFG prepared with 20 wt\% WPI and (a) 5 vol\% oil, (b) 10 vol\% oil, (c) 15 vol\% oil, and (d) 20 vol\% oil.

particles appear to act as hard spheres, as such deformation does not occur which is reflected by the lower stresses required to induce flow, ${ }^{39}$ as shown in Fig. 7. Here it is possible to see a similar correlation as observed for the frequency sweeps, where a plateau is reached for oil fractions above $10 \mathrm{vol} \%$. This yielding insensitivity towards increasing oil fractions again infers a network of deformed particles closely packed together, as previously suggested. ${ }^{39}$

3.2.3. Suspension flow behaviour. System flow behaviours for all WPI EmFG were studied and presented in Fig. 8. EmFG showed marked shear thinning behaviours typical of highly flocculated suspensions. ${ }^{40}$ At very low shear rates $\left(\sim 0.01 \mathrm{~s}^{-1}\right)$ an apparent shear thickening can be observed as a resultant effect of soft jamming. Continued increase in the applied strain resulted in thinning of the suspensions. The observed thinning is due to the inhomogeneous flow across the shear profile applied, as a result of the break down to the weakly flocculated network. ${ }^{40}$ Thus a degree of inter-particle interactions is suggested, where initially clusters of mesostructures are broken down to form single mesostructures (smaller flocs) and eventually microstructures (single particles). To analyse this further, data has been presented for the ramp up, down and additionally a second sweep taken immediately after the first.

The presence of hysteresis highlighted a thixotropic nature arising through the breakdown of floccules and inter-particle interactions, as seen in Fig. 9. The plot shows a similar correlation as previously observed for yield stresses and frequency sweeps whereby the highly packed systems ( 10 to $20 \mathrm{vol} \%$ oil) have a greater hysteresis. This indicates that jammed systems have a much higher degree of inter-particle interactions as a result of greater packing arising through particle deformation. As expected, where the particles remain unjammed a lower degree of thixotropy is observed, as non-deformed particles

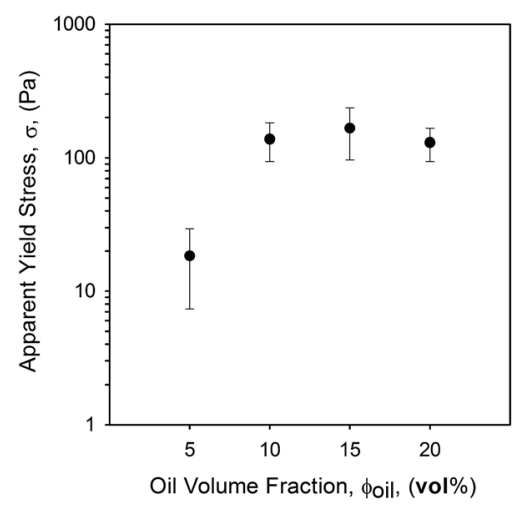

Fig. 7 Apparent yield stresses determined using stress sweeps, as the point at which the storage modulus $\left(G^{\prime}\right)$ cross the loss modulus $\left(G^{\prime \prime}\right)$ for EmFG systems prepared using $20 \mathrm{wt} \% \mathrm{WPI}$ and oil fractions between 5 and 20 vol\%. 
(a)

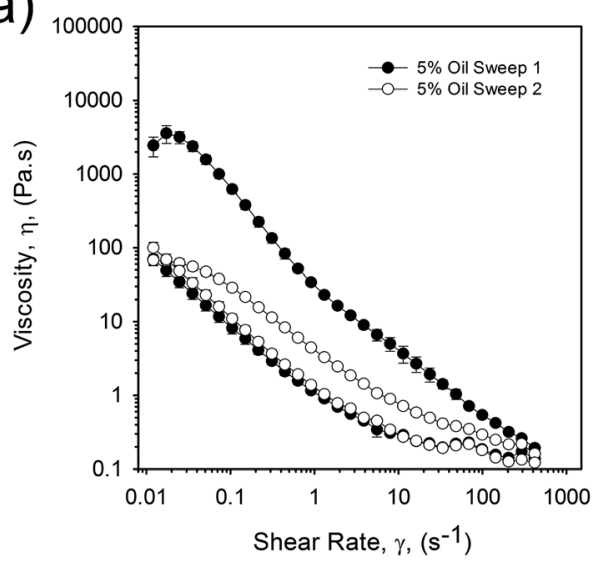

(c)

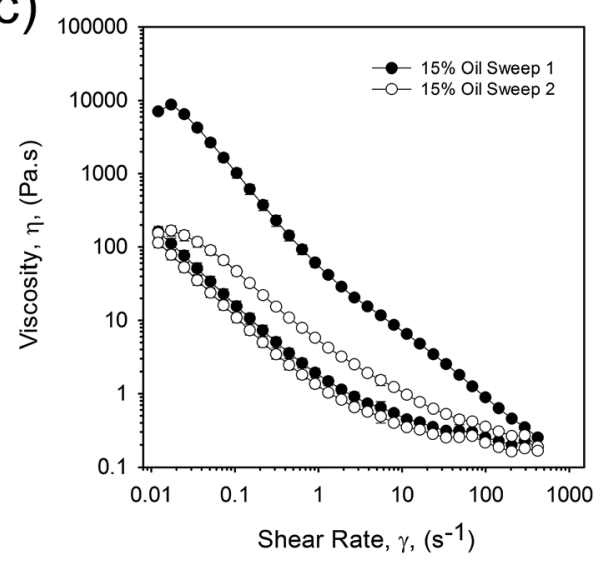

(b)

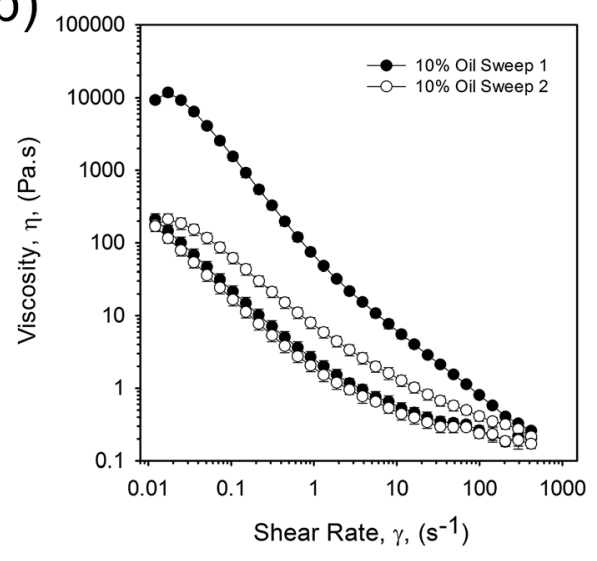

(d)

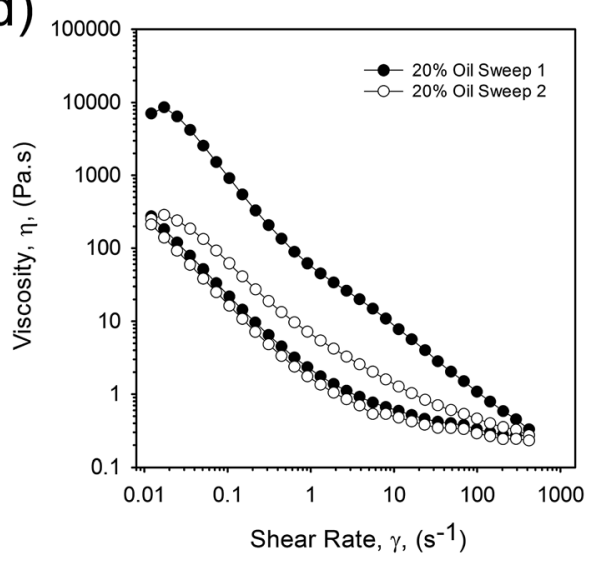

Fig. 8 Viscosity profiles obtained for 20 wt\% WPI EmFG containing (a) 5 vol\% oil, (b) 10 vol\% oil, (c) 15 vol\% oil, and (d) 20 vol\% oil. Closed markers represent sweep 1 and open, sweep 2.

present a smaller surface area for inter-particle interactions to occur. However, data obtained for the second sweep showed a similar thixotropic behaviour for all systems, irrespective of oil content. Additionally, a combination of microscopy and light scattering techniques showed that the applied shear was insufficient to break the single particles, with the same particle size distributions observed pre and post shear sweeps (data not shown). As such it is argued that the shear applied throughout the first sweep disrupts the network to an extent that all particles behave as independent spheres. This is followed by restructuring between the particles, but on a scale that is much slower than the break down.

3.2.4. Material recovery. The recovery was further probed using oscillatory rheology. Primarily the system underwent rejuvenation, whereby the flocculated structure is broken down at a shear rate found within the shear-thinning region for all systems $\left(10 \mathrm{~s}^{-1}\right.$ for $\left.10 \mathrm{~s}\right)$. The structuring was then observed through the storage modulus $\left(G^{\prime}\right)$ over the subsequent $30 \mathrm{~min}$, Fig. 10. The recovery curves show a two-step process, initially rapid, followed by a more gradual increase in elastic modulus, observed as a power function $(0.45 \pm 0.7)$. The same power law dependency observed across all systems can be argued as the same recovery mechanism being observed i.e. initially there is rapid formation of a large number of small flocs, as the flocs grow in size the change in $G^{\prime}$ slows as fewer larger particles are available to interact. The extent and rate of recovery however was dependent on the volume fraction of the particles. Volume fractions greater than 0.64 could not be significantly differentiated, with the extent and rate of recovery depending on the self-similarity of the

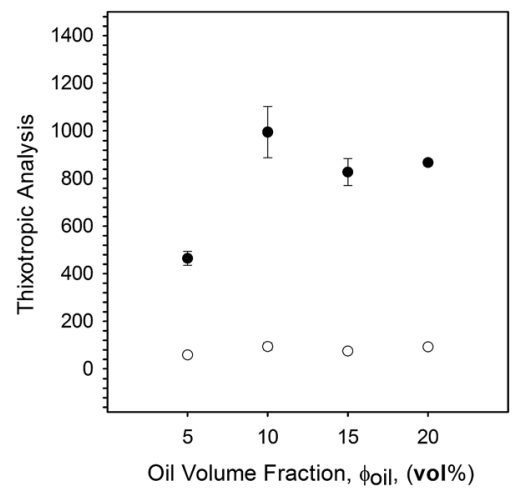

Fig. 9 Thixotropic analysis obtained as the area between the viscosity profiles for 20 wt\% WPI EmFG with increasing oil phase volumes from 5 to 20 vol\% $((\mathbf{)})$ denote thixotropic analysis for sweep 1 and $(\bigcirc)$ denotes values for sweep 2). 


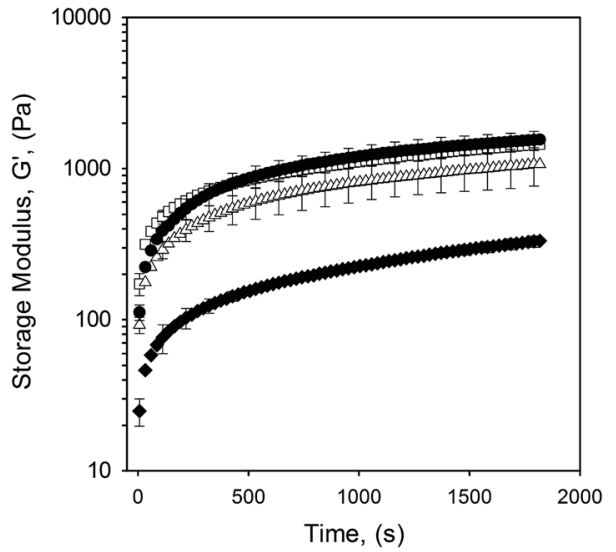

Fig. 10 Storage modulus as a function of time for EmFG systems ( $\bullet$ ) 5 vol\% oil, $(\square) 10$ vol\% oil, $(\triangle) 15$ vol\% oil, and $(\bullet) 20$ vol\% oil. All EmFG underwent a rejuvenation process of $10 \mathrm{~s}^{-1}$ for $10 \mathrm{~s}$ before measuring $G^{\prime}$ at $1 \mathrm{~Hz}$ and $1 \mathrm{~Pa}$ stress for 30 minutes.

shells. For EmFG $<0.64, G^{\prime}$ values were lower by a factor of 10 ; as is observed from the frequency sweep data, again due to much less interacting where particles are further apart in space. As such, the hysteresis observed through dynamic shear experiments is suggested to arise not through the rupturing of a weakly gelled structure, but from the break down of reversible interactions between the particles.

\section{Conclusions}

This study has shown that oil droplets can be incorporated into a WPI gel layer by applying a shear-gel technique. Entrapment efficiency was observed to be dependant on the protein concentration, as a function of the viscosity and flow behaviours, reaching up to 99\% encapsulation. By applying shear during the WPI sol-gel transition discreet micron sized spherical capsules were obtained, with enhanced structuring properties. Small deformation rheology was used to characterise the suspensions, which showed pseudosolid like behaviour at rest, however, by applying a shear force to the system that is greater than the yield stress, the suspensions could be made to flow. Suspension rheology highlighted a significant dependence on the oil fraction, with the addition of oil increasing the effective phase volume of the particles, resulting in an increase in particle proximity. Increasing the oil content to around $10 \mathrm{vol} \%$ led to packing fractions that exceeded those for hard spheres. As such, particle properties have been expressed as soft and deformable. Flow behaviours of the suspensions were indicative of highly flocculated systems, where marked shear thinning was observed through the break up of weakly aggregated flocs and mesostructures. Furthermore, when left under quiescent conditions the particulate suspensions showed significant recovery, displaying the occurrence of reversible interactions.

\section{Acknowledgements}

The authors would like to thank the sponsoring bodies including EPSRC, Kerry Group, and also Birmingham Science
City: Innovative Uses for Advanced Materials in the Modern World (West Midlands Centre for Advanced Materials Project 2), with support from Advantage West Midlands (AWM) and part funded by the European Regional Development Fun (ERDF), for the use of the confocal microscope used in this research.

\section{References}

1 I. T. Norton, D. A. Jarvis and T. J. Foster, Int. J. Biol. Macromol., 1999, 26, 255-261.

2 N. Altmann, J. J. Cooper-White, D. E. Dunstan and J. R. Stokes, J. Non-Newtonian Fluid Mech., 2004, 124, 129136.

3 J. E. Norton and I. T. Norton, Soft Matter, 2010, 6, 3735-3742.

4 I. Fernández Farrés, R. J. A. Moakes and I. T. Norton, Food Hydrocolloids, 2014, 42(3), 362-372.

5 C. L. A. Berli and D. Quemada, Langmuir, 2000, 16, 79687974.

6 D. A. Garrec, B. Guthrie and I. T. Norton, Food Hydrocolloids, 2013, 33, 151-159.

7 I. Fernández Farrés, M. Douaire and I. T. Norton, Food Hydrocolloids, 2013, 32, 115-122.

8 A. Gabriele, F. Spyropoulos and I. T. Norton, Food Hydrocolloids, 2009, 23, 2054-2061.

9 S. Adams, W. J. Frith and J. R. Stokes, J. Rheol., 2004, 48, 1195.

10 E. Çakır and E. A. Foegeding, Food Hydrocolloids, 2011, 25, 1538-1546.

11 S. M. Fitzsimons, D. M. Mulvihill and E. R. Morris, Food Hydrocolloids, 2008, 22, 485-491.

12 K. Nishinari, H. Zhang and S. Ikeda, Curr. Opin. Colloid Interface Sci., 2000, 5, 195-201.

13 I. T. Norton and W. J. Frith, Food Hydrocolloids, 2001, 15, 543-553.

14 C. Rocha, J. A. Teixeira, L. Hilliou, P. Sampaio and M. P. Gonçalves, Food Hydrocolloids, 2009, 23, 1734-1745.

15 T. van Vliet, Colloid Polym. Sci., 1988, 266, 518-524.

16 D. B. Genovese, Adv. Colloid Interface Sci., 2012, 171-172, 116.

17 E. Dickinson and J. Chen, J. Dispersion Sci. Technol., 1999, 20, 197-213.

18 E. Dickinson, J. Chem. Soc., Faraday Trans., 1998, 94, 16571669.

19 E. Dickinson and Y. Matsumura, Int. J. Biol. Macromol., 1991, 13, 26-30.

20 D. Sağlam, P. Venema, R. de Vries, L. M. C. Sagis and E. van der Linden, Food Hydrocolloids, 2011, 25, 1139-1148.

21 A. Sullo, R. L. Watson and I. T. Norton, in Gums and Stabilisers for the Food Industry 17: The Changing Face of Food Manufacture: The Role of Hydrocolloids, The Royal Society of Chemistry, 2014, pp. 287-302.

22 D. A. Garrec and I. T. Norton, J. Food Eng., 2012, 112, 175182.

23 R. J. A. Moakes, A. Sullo and I. T. Norton, Food Hydrocolloids, 2015, 45, 227-235.

24 C. Chung, B. Degner, E. A. Decker and D. J. McClements, Innovative Food Sci. Emerging Technol., 2013, 20, 324-334. 
25 A. Matalanis and D. J. McClements, Food Hydrocolloids, 2013, 31, 15-25.

26 Z. Zhang, R. Zhang, E. A. Decker and D. J. McClements, Food Hydrocolloids, 2015, 44, 345-352.

27 A. I. Romoscanu and R. Mezzenga, Langmuir, 2006, 22, 78127818.

28 M. Stading and A.-M. Hermansson, Food Hydrocolloids, 1990, 4, 121-135.

29 P. Walkenstrom, E. Windhab and A.-M. Hermansson, Food Hydrocolloids, 1998, 12, 459-468.

30 A. M. Hermansson, J. Texture Stud., 1975, 5, 425-439.

31 M. A. M. Hoffmann, S. P. F. M. Roefs, M. Verheul, P. J. J. M. van Mil and C. G. de Kruif, J. Dairy Res., 1996, 63, 423-440.
32 W. H. Sawyer, J. Dairy Sci., 1968, 51, 323-329.

33 D. J. McClements, Food Emulsions Principles, Practices, and Techniques, CRC Press, 2005.

34 P. Walstra, Chem. Eng. Sci., 1993, 48, 333-349.

35 S. B. Ross-Murphy, Polym. Gels Networks, 1994, 2, 229-237.

36 I. M. Krieger and T. J. Dougherty, J. Rheol., 1959, 3, 137-152.

37 N. Koumakis, A. Pamvouxoglou, A. S. Poulos and G. Petekidis, Soft Matter, 2012, 8, 4271-4284.

38 J. Chen, E. Dickinson, M. Langton and A.-M. Hermansson, LWT-Food Sci. Technol., 2000, 33, 299-307.

39 A. P. le Grand and G. Petekidis, Rheol. Acta, 2008, 47, 579590.

40 D. Quemada, Eur. Phys. J.: Appl. Phys., 1998, 1, 119-127. 